\title{
Introduction to the special issue on Immunology at Mount Sinai
}

\author{
Lloyd Mayer $\cdot$ Sergio Lira $\cdot$ Adrian Ting • \\ Patricia Cortes
}

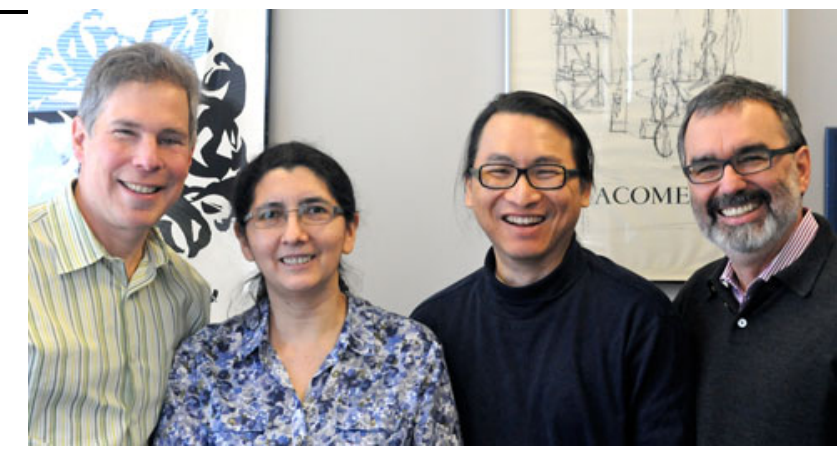

Lloyd Mayer Patricia Cortes Adrian Ting Sergio Lira

The history of modern immunology at Mount Sinai dates back to 1985, when the then dean Nathan Kase decided to expand our efforts in the area. At that point, we had only one faculty member at Mount Sinai whose research focus was in immunology, Constantin Bona in the Department of Microbiology. In the next few years, Lloyd Mayer, Jay Unkeless, Charlotte Cunningham-Rundles, Wayne Yokoyama, Paul Klotman, Tom Moran, Karen Zier, and Terry Davies were recruited. Each developed his/her own independent research program with institutional and departmental support. The problem remained that these investigators were housed in separate departments (Microbiology, Medicine, and Biochemistry), and graduate students were identified within these other disciplines and not in Immunology per se. In 1994, the Immunobiology program was given status as an independent area of concentration. Graduate students could now declare themselves as Immunology students with specific course work requirements. What evolved was a course in Fundamentals of Immunobiology (a 24-27 h course), an Advanced Topics in Immunology ( 3 separate $14 \mathrm{~h}$ interactive seminar series/year relating to new areas of interest taught by the Immunology faculty) and an Immunology Journal Club. The students were also required to participate in the weekly Immunology seminar series as well as in the work-inprogress meetings.

The glue that held the program together, even before its independent status as a graduate program, was a program project grant in autoimmunity with Constantin Bona, MD, $\mathrm{Ph} . \mathrm{D}$. as the principal investigator. This PPG was funded

L. Mayer $(\bowtie) \cdot$ S. Lira $\cdot$ A. Ting $\cdot$ P. Cortes

Immunology Institute, Mount Sinai Medical Center, 1425 Madison Avenue, New York, NY 10029, USA e-mail: lloyd.mayer@mssm.edu from 1987 to 2003 and fostered many collaborations, the Flow Cytometry Core, the Hybridoma Core, the Immunology seminar series, work-in-progress meetings, the journal club and, most importantly, many new interactive program grants.

In 1997, the Immunobiology Center was officially formed with Lloyd Mayer as the Chairman. This center was given departmental status with its own appointments and promotions power, an independent budget, and seed money to be used for the recruitment of between 5 and 7 new faculty members (Assistant/Associate Professor level). The mission of this new center was to expand and build upon the existing strength in Immunology at Mount Sinai. The center was provided state of the art laboratory research space in the Icahn Building, resources for recruitment, and a sizeable equipment budget. The Center grew from an initial 14 faculty (primary and secondary) to 25 . With the new Dean came a switch in focus, from Departments to Institutes.

The Immunology Institute of Mount Sinai School of Medicine was established in February of 2007 with Sergio Lira and Lloyd Mayer as Co-Directors. This new initiative included 37 investigators at the Sinai campus and its main mission is to foster research, collaboration, and education in immunology. With the establishment of the Institute, we also created a $\mathrm{MD} / \mathrm{PhD}$ and $\mathrm{PhD}$ program in Immunology. For our educational mission, scientists meet weekly and discuss their findings at the Work-in-progress Seminar Series; once a month, we host internationally renowned immunologists at the Immunology Seminar Series, and once a year, we hold an Institute retreat to bring together all of the laboratories. The goal remains to promote a highly interactive productive environment for the training of graduate students, postdoctoral fellows, junior and senior faculty. Our recruitments over the past 15 years have 
already reaped benefits in terms of creating a sense of community and an interactive training environment. In fact, our faculty meetings have become sessions where the faculty present their most novel and innovative work, looking to foster collaborations. This has been a very successful approach as both the immunodeficiency PPG and mucosal immunology/IBD PPG arose from these meetings. They have solidified Immunology as a distinct entity that is easily recognized by students and postdoctoral fellows as a desirable area for training. Recruitment of students has increased tremendously, with the pool of applicants doubling in the last 2 consecutive years. Furthermore, we have a training grant for over 8 years that funds postdoctoral fellows and graduate students. In addition to our graduate program, that focuses on the training of PhDs and MSTP students, our faculty maintains a strong commitment to the mentoring of medical students, residents, and clinical fellows.

As we grew larger, we reached into other areas of the immune system and expanded the existing ones, including primary immunodeficiency (Charlotte Cunningham-Rundles), food allergy disorders (Hugh Sampson), cancer biology (Steven Burakoff), genetic models of human diseases (Sergio Lira), transplantation (Peter Heeger, Barbara Murphy) and mucosal immunity/IBD (Lloyd Mayer). Each area had the same interactive nature and fostered many PPGs and $\mathrm{U}$ grants. Each area had a focused clinical interest, with a specific patient population. These combined activities have increased the number of research collaborations, which have led to funding of several program grants.

The role of the immune system in disease processes can be grouped into five general areas: inflammation, immunoregulation, immunocompetence, autoimmunity, and mechanisms of infection.

Research in inflammation focuses on multiple areas. Investigators study the ontogeny and composition of immune cell populations (Merad, Mayer, Alexandropoulos), cellular migration (Lira, Furtado, Esplugues), signaling (Ting, Chen, Xiong, Yeretssian), apoptosis and necrosis (Blander, Ting, Yeretssian), and inflammatory myopathy and fibrosis (Zhou).

In the area of immunoregulation, we study the function of specific suppressor or regulatory pathways (Mayer, Sikora, Chen), role of complement in $\mathrm{T}$ cell function and transplantation (Heeger) and the role of microRNAs in immune cell function (Brown). These are emphasized translationally by several human disorders.

Inflammatory bowel diseases Mount Sinai's research and clinical preeminence in inflammatory bowel disease has been a major strength of our program. Mount Sinai and IBD have been synonymous since 1932 (initial description of Crohn's disease at Mount Sinai). There is a strong base of clinical and basic research and probably one of the largest patient populations in the world. This access to patients allows us to perform unique studies looking at the control of mucosal inflammation (Mayer), lymphocyte/ epithelial cell interactions (Dahan, Berin, and Mayer) and the microbiome.

Funding for this center has been provided by a program grant in innate and adaptive immunity in IBD (Mayer, Lira, Blander, Xiong), several RO1s, and a generous gift from the Helmsley Foundation that has allowed us to further recruit into the center (Cerutti, Espluges, Yeretssian).

Primary immunodeficiency Investigators focus on basic aspects of B cell function (Cortes, Cerutti, CunninghamRundles), and we have an outstanding clinic in Primary Immunodeficiency. Charlotte Cunningham-Rundles runs the largest Primary Immunodeficiency clinic in the Northeast (probably the US). In addition to a program grant and several RO1s, there are several ongoing clinical trials.

Food allergy Hugh Sampson runs the pediatric food allergy clinic, although some adults are seen as well. Clinical research, clinical trials, and basic research are all ongoing and well funded. Many newer approaches are first tested here. Adult food allergy is a less-studied disorder. Cecilia Berin studies the role of physiologic or environmental adjuvants that may promote the development of allergic sensitization by altering the phenotype of mucosal dendritic cells and Paula Busse studies mechanisms of allergies in older populations. Xu-Min $\mathrm{Li}$ and Madhu Masilamani study immunopathogenic mechanisms underlying food allergy and novel therapies for these allergic diseases. The results of these efforts are a well-funded program in food allergy (Sampson, Mayer and Berin).

Tumor immunology We recently recruited Steven Burakoff to head our efforts in cancer biology. The cancer center has enjoyed tremendous growth recruiting worldclass clinicians, clinical researchers as well as basic scientists. Some of the investigators focus on the role of inflammation in cancer (Merad, Lira, Burakoff, Sikora, Chen, Palucka, Blander, Xiong) while others assess immune responses to tumors with an eye in therapeutic intervention (Sikora, Chen, Palucka, Brody).

Autoimmunity Autoimmunity is defined as an abnormal immune response against self. While the basic definition is still valid, it has been changed over the past decade as our understanding of immunoregulatory networks has improved. What has been recognized is that reactivity to self is not always abnormal and that frequently what starts as a focused response against a foreign antigen can eventuate in the development of an autoimmune disease. It has also led to the redefinition of a series of chronic 
inflammatory disorders where the response is not directed specifically at self but tissue destruction occurs as a result of an uncontrolled immune reaction. Furthermore, it is now appreciated that B cells are not the only driving force in autoimmunity. Many diseases reflect aberrant $\mathrm{T}$ cell responses and many of the previously thought of B-cellmediated disorders have a basis in T cell dysfunction. As in any immune response, antigen uptake and presentation are initiating factors in autoimmunity. The presence or absence of co-stimulation affects the nature of the response. However, the role that cytokines play in amplifying the response is becoming increasingly evident. Novel strategies designed at targeting cytokines, their receptors or their signaling pathways have been developed over the past 10 years. Several of these strategies have proven valuable in the control of previously unmanageable disease.

Research on basic autoimmune mechanisms is being carried by several labs in the Institute. The labs focus on central mechanisms of selection (Alexandropoulos), ectopic lymphoid neogenesis in autoimmune diseases of pancreas and thyroid (Lira, Furtado, Tomer and Davies), dysregulated chemokine signaling (Diaz), mechanisms of tolerance (Mayer, Ochando, Heeger). Sinai has strong programs in thyroid autoimmune disease (Terry Davies and Yaron Tomer) and in Type I diabetes (Tomer, Brown, Lira) and transplantation (Murphy, Heeger, Ochando).

Mechanisms of infections Mechanisms of infections are also an important area of concentration for our faculty. The Chen laboratory explores mechanisms that govern cell-tocell transmission of HIV through the virological synapses and responses to flu virus infection are investigated by the Moran laboratory. Computational approaches are use to integrate and further understand host responses to viral infection (Sealfon). In addition, we have numerous interactions with our colleagues from the department of

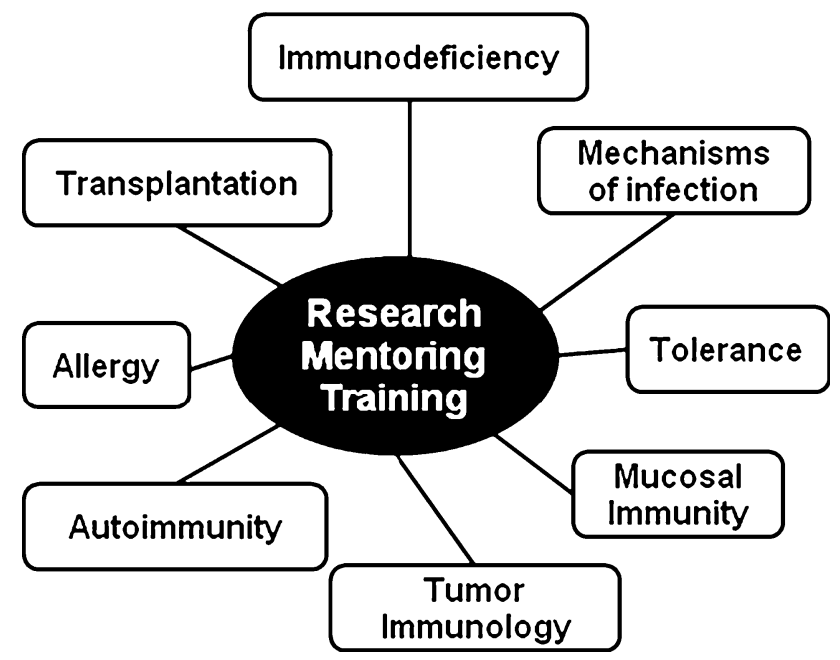

Fig. 1 Immunology programs at Mount Sinai

Microbiology that also participates in the training of our students. Their work in systems such as dengue virus (Sesma), neurotropic flavivirus (Lim), and cytomegalovirus (Tortorella) among others, enriches the collaborations and training opportunities for the immunology community.

All in all, the Immunology Institute comprises a focused interest in human disease, mechanisms of disease pathogenesis, and therapeutic interventions. We have unique patient populations who give us an advantage in terms of translational science. Where we do not have direct access to human samples, animal models of disease are created. This melding of patient populations with animal models and multiple approaches to study mechanisms is unique and serves to distinguish the Immunology Institute from other Immunology programs around the country. In our institute, faculty with interest in diverse aspects of the immune system works together to achieve excellence in research, mentoring, and training (Fig. 1). 